\title{
Manejo deglutorio del adulto mayor institucionalizado por parte de técnicos en enfermería: un estudio cualitativo
}

Swallowing Management of Institutionalized Older Adults by Nursing Technicians:

\section{A Qualitative Study}

Manejo deglutório do idoso institucionalizado por parte de técnicos em enfermagem:

um estudo qualitativo

Felipe Henríquez, Fgo., Mg., PhD ${ }^{1,2}$

Jordan Ferrufino, Lic. ${ }^{1,3}$

Nicol Inzunza, Lic. ${ }^{1,4}$

Ana Nancucheo, Lic. ${ }^{1,5}$

Eduardo Padilla, Lic. ${ }^{1,6}$

Valeska Soldán, Lic.1,7

Camilo Morales, MSc., Lic.,**

1 Departamento de Procesos Terapéuticos, Facultad Ciencias de la Salud, Universidad Católica de Temuco, Temuco (Chile). orciD: https://orcid.org/0000-0002-7980-2844

Autor de correspondencia: camilo.morales@uct.cl orciD: https://orcid.org/0000-0002-5811-2908

2 ORCID: https://orcid.org/0000-0002-7980-2844

3 ORCID: https://orcid.org/0000-0003-1067-5696

4 ORCID: https://orcid.org/0000-0003-3779-1494

5 ORCID: https://orcid.org/0000-0002-7117-6981

6 ORCID: https://orcid.org/0000-0002-9132-4187

7 ORCID: https://orcid.org/0000-0003-2500-8706 


\section{Resumen}

Introducción: se busca profundizar acerca del manejo cotidiano de las complicaciones de la deglución en centros del adulto mayor, las cuales generalmente asume el técnico o auxiliar de enfermería (TENs), quienes son los encargados de su alimentación. Materiales y métodos: se llevó a cabo un estudio con enfoque cualitativo basado en la teoría fundamentada. Se realizaron grupos de discusión y entrevistas en profundidad a dieciocho tens de tres establecimientos de larga estadía en la Araucanía (Chile). Se analizaron los datos a través de codificación abierta y axial, con los cuales se obtuvieron seis categorías analíticas sobre esta situación. Resultados: los TENs y los auxiliares de la alimentación reportaron desconocimiento del uso y pertinencia de estas; además, comentaron que dichas maniobras las aprendieron mediante la observación a otros profesionales itinerantes dentro del centro, y no producto de la formación o trabajo interdisciplinario. Conclusión: dado el contexto y las limitaciones derivadas de la formación de los TENs, surge la necesidad de incrementar sus conocimientos y mejorar sus prácticas relacionadas con la deglución y alimentación, con el objetivo de disminuir el riesgo de ocurrencia de situaciones que agraven la salud de los adultos mayores. Se discute, así mismo, la pertinencia de que estos centros cuenten con profesionales capacitados en cuanto al abordaje de los trastornos de la deglución, pues ello contribuirá a mejorar este aspecto, en función del trabajo coordinado con el resto del equipo.

Palabras clave: trastornos de deglución; alimentación; anciano; paramédico.

\section{Abstract}

Introduction: This study examines the daily management of swallowing complications of older adults in centers, which generally assumes that the technician or auxiliary nurses (TENs) are the ones in charge of their feeding. Materials and Methods: A study was conducted with a qualitative approach based on the grounded theory. A focus group and in-depth interviews were performed with 18 nursing technicians and assistants from 3 long-stay facilities in Araucanía, Chile. Data were analyzed through open and axial coding, obtaining 6 analytical categories on this situation. Results: The teNs and auxiliary feeding reported to have no knowledge of the use and relevance of these maneuvers and reported that these were learned via the observation of other traveling professionals within the center and were not a product of interdisciplinary training. Conclusion: Given the context and the limitations derived from the formation of TENS, there is the need to increase their knowledge and improve their practices related to swallowing and feeding, with the aim of reducing the risk of occurrence of situations that aggravate the health of older adults. The relevance of these centers incorporating qualified professionals in the management of swallowing disorders, who would also contribute to improving these aspects, based on coordinated work with the rest of the team was also discussed.

Keywords: Deglutition disorders; feeding; aged; paramedic.

\section{Resumo}

Introdução: se busca aprofundar acerca do manejo cotidiano das complicações da deglução em centros do idoso, os quais geralmente os assume o técnico ou auxiliar de enfermagem (TENs) os quais são os encarregados de sua alimentação. Materiais e métodos: levou-se a cabo um estudo com enfoque qualitativo baseado na teoria fundamentada. Se realizaram focus group e entrevistas em profundidade a 18 TENs de 3 estabelecimentos de longa estadia em Auracanía, Chile. Se analisaram os dados através de codificação aberta e axial, obtendo-se 6 categorias analíticas sobre esta situação. Resultados: os TENs e auxiliares da alimentação reportaram desconhecimento do uso e pertinência destas, comentando para além de ditas manobras foram aprendidas através da observação a outros profissionais itinerantes dentro do centro, e não produto da formação ou trabalho interdisciplinar. Conclusão: devido ao contexto e às limitações derivadas da formação dos TENs, surge a necessidade de incrementar seus conhecimentos e melhorar suas práticas relacionadas à deglução e alimentação, com o objetivo de diminuir o risco de ocorrência de 
situações que agravem a saúde dos idosos. Se discute para além da pertinência de que estes centros contem com profissionais idóneos em quanto à abordagem dos transtornos da deglução, que contribuíram também a melhorar estes aspectos, em função do trabalho coordenado com o resto da equipe.

Palavras-chave: transtornos de deglução; alimentação; idoso; paramédico.

\section{Introducción}

T a presbifagia constituye una adaptación gradual de la deglución, consecuencia de los Lcambios producto de la edad. En ese sentido, se ven afectadas la fase oral preparatoria, la oral, la faríngea e incluso la fase esofágica (1). Sin embargo, no solo las modificaciones producto de la edad pueden dificultar este proceso, sino que también pueden existir afectaciones neurológicas, como secuelas de accidente cerebrovascular, traumatismo encefalocraneano, demencias o procesos neurodegenerativos, entre otros, los cuales pueden generar alteraciones de esta índole, que en dichos casos se denomina disfagia, que denota una dificultad o disconfort para transferir el bolo alimenticio desde la boca hacia el estómago (2). Desde el punto de vista anatómico, la disfagia puede deberse a disfunciones orofaríngeas o esofágicas, y desde la mirada fisiopatológica, a causas estructurales o funcionales como las ya mencionadas (3). Como posibles consecuencias existen tres grandes complicaciones: respiratorias (al desencadenarse patologías como neumonías aspirativas), alimenticionutricionales (alteraciones en una o más fases de la deglución, disminución en el peso o decaimiento del aporte nutricional) y en la calidad de vida (al apartarse la persona de círculos sociales en momentos cuando se deba compartir o alimentar). Para evitar esta situación en el usuario, se deben adoptar una serie de modificaciones en los alimentos y bebidas para asegurar una correcta nutrición e hidratación, pues ello permitirá mantener un adecuado estado de salud (4).

Cuando existen dificultades en el proceso de la alimentación, el fonoaudiólogo debe realizar la evaluación correspondiente y dejar consignada la rehabilitación y, según cada caso, la implementación de maniobras compensatorias de alimentación, las cuales consisten en modificaciones posturales (cambios de posición para favorecer el paso del bolo por áreas orofaríngeas más conservadas), modificación de la consistencia de los alimentos (tipo néctar, miel o pudín, por ejemplo) o cambios en la velocidad de entrega, volumen y técnicas de incremento sensorial (estimulación termotáctil en pilares faríngeos, cambios en la temperatura del bolo, aplicación de sabores ácidos), entre otros, con el objetivo de mantener una alimentación saludable, sana y segura en el usuario presbifágico o disfágico $(5,6)$.

Estas maniobras compensatorias debería indicarlas un fonoaudiólogo capacitado y enseñarlas a profesionales con formación en geriatría o familiares para aplicarlas en diferentes contextos (hospitalario, salud primaria, domiciliario, establecimientos de larga 
estadía, etc.) (7). Por tanto, se deberían explicar también a técnicos en enfermería, a auxiliares en enfermería o a quien corresponda para su posterior administración en adultos mayores presentes en contextos clínicos, hogares o los llamados establecimientos de larga estadía del adulto mayor (Eleam). De particular importancia es el último, debido a que, en dichos contextos, son los técnicos quienes proveen cuidados y pasan la mayoría del tiempo con los adultos mayores.

Se define Eleam como aquel establecimiento donde residen personas de sesenta años de edad o más, quienes por motivos biológicos, psicológicos o sociales requieren un ambiente acorde con sus necesidades y cuidados diferenciados, dentro de los que se incluyen la prevención, la mantención y la estimulación de su salud y funcionalidad (8). Esto surge como respuesta a los nuevos desafíos que impone el envejecimiento local y general latinoamericano, pues se intenta tomar en cuenta la heterogeneidad de los adultos mayores, sus necesidades y el reconocimiento sobre su contribución a la sociedad (9).

En Chile, por normativa, el auxiliar en enfermería con mención de geriatría es el encargado de llevar a cabo la alimentación de los usuarios que pertenecen al Eleam (10). Sin embargo, en esta no se explicita si sus acciones deberían estar dirigidas a personas que poseen o no trastornos de la deglución o de la alimentación, pese a que en los protocolos de estos establecimientos se especifica la necesidad de manejarlos (11).

Según las mallas curriculares de los establecimientos formadores, dentro de los conocimientos técnicos que deberían manejar los profesionales para los cuidados sobre la alimentación en los Eleam se encuentran conocimientos generales de anatomía, fisiología y neurología de la deglución; disfagia, y clasificación de los trastornos de la deglución (12-14). Por otro lado, el detalle de las funciones que es capaz de realizar el técnico en enfermería, enfermero o auxiliar de enfermería se relaciona con el manejo de síndromes y tratamientos como incontinencia, alimentación normal, constipación, dolor, alimentación por sonda nasogástrica, manejo vía venosa, úlceras por presión, oxigenoterapia, entre otros, sin considerar el manejo deglutorio (11).

Actualmente, pocos estudios han aludido al conocimiento formal que tienen o deberían tener sobre estos aspectos los técnicos en enfermería con mención en geriatría; como principales resultados se han hallado dificultades relacionadas con la falta de información en su proceso formativo o de estrategias utilizables en estos contextos y problemas en la identificación de sintomatología asociada con los trastornos de la deglución (15-18).

En Chile, solamente un estudio hace referencia a las competencias clínicas en el manejo hospitalario de estos usuarios, si es que realizan estas maniobras en pacientes con dificultades alimentarias y de qué forma ven el impacto de estas en la calidad de vida relacionada con la deglución de las personas a quienes tratan (19). 
Entonces, tomando en cuenta que ellos son los miembros del equipo quienes se encuentran en la cotidianeidad de los adultos mayores, especialmente en los contextos domiciliario y en Eleam, además de la relevancia de una buena ejecución de este rol para la calidad de vida de los adultos mayores, el objetivo de esta investigación consistió en determinar si los técnicos en enfermería y auxiliares en enfermería (TENS) conocen maniobras compensatorias de alimentación en adultos mayores con presbifagia o disfagia en este tipo de establecimientos, a fin de profundizar en sus percepciones, opiniones y experiencias sobre dicha situación.

\section{Materiales y métodos}

$\mathrm{L}$ a investigación se enmarca dentro de un paradigma cualitativo de investigación, con un $\boldsymbol{J}$ diseño de teoría fundamentada que pretende generar conocimientos sobre temas poco explorados. Genera conceptos explicativos de un fenómeno a partir de las visiones de los participantes, situados en un contexto específico (20). A la hora de seleccionar a los participantes, se tuvo en cuenta, como criterio de inclusión, que ejercieran un rol de cuidador/a de adultos mayores en Eleam, ya sea técnicos o auxiliares en enfermería. Se llevó a cabo un muestreo intencionado o por conveniencia, accediendo en total a dieciocho teNs dentro de los Eleam Años Mejores, Vicente de Paul y Las Araucarias, los cuales cuentan, además, con enfermeros, kinesiólogos, nutricionistas y terapeutas ocupacionales. Estos centros pertenecen a la región de la Araucanía (Chile) (21).

El estudio contó con la aprobación de Comité de Ética de la Universidad Católica de Temuco (Chile), con resolución 97/2016, y con la autorización de los directivos de los Eleam Años Mejores, Vicente de Paul y Las Araucarias, respetando los principios éticos dela Declaración de Helsinki(22). Posterior a ello, se llevó a cabo el reclutamiento, en el que se les explicaron a los tens los procedimientos, los riesgos y los beneficios del estudio. También se dieron a conocer los roles de los integrantes del equipo de investigación en la ejecución de esta. Cada uno de los participantes acordó su participación mediante la firma de un consentimiento informado.

La información se recopiló mediante grupos de discusión (45 minutos en promedio) y entrevistas en profundidad (30 minutos en promedio), los cuales se llevaron a cabo en los salones comunes de los mismos establecimientos y en una sala aislada, respectivamente (23). Estos se grabaron previa autorización de los participantes y se guiaron con una pauta de preguntas abiertas y de posibles temas que pudieran surgir; posteriormente, se transcribieron las respuestas. La información obtenida la analizaron dos personas de manera separada y luego fue consensuada, mediante el proceso de codificación abierta, axial y generación e interacción de 
categorías, a través del método de comparación constante, de acuerdo con los procedimientos propuestos por la teoría fundamentada (20).

La transcripción literal de los grupos de discusión y de las entrevistas en profundidad, además del análisis de los resultados, se presentó a los participantes para corroborar su veracidad. Las tablas 1, 2 y 3 resumen las características de la población de estudio y el esquema de preguntas entrevista semiestructurada y grupos de discusión, respectivamente.

Tabla 1. Caracterización de los participantes

\begin{tabular}{lccc}
\hline & & n & \% \\
\hline & Género & & \\
\hline Femenino & & 17 & 94 \\
Masculino & & 1 & 6 \\
\hline & Establecimiento & \\
\hline Eleam Años Mejores & & 6 & 33.3 \\
Eleam Las Araucarias & & 7 & 38.9 \\
Eleam Vicente de Paul & & 5 & 27.8 \\
\hline & Formación & & \\
\hline Educación universitaria & & 1 & 5.6 \\
Técnico nivel superior & & 7 & 38.9 \\
Técnico nivel medio & & 10 & 55.5 \\
Edad (promedio \pm DE) & & 36.4 & $( \pm 12.1)$ \\
Años de experiencia (promedio \pm DE) & 9.61 & $( \pm 8.3)$ \\
\hline
\end{tabular}

DE: desviación estándar.

Tabla 2. Resumen de preguntas de la entrevista en profundidad

\section{Preguntas}

1. ¿Qué conocimientos tiene acerca del concepto de deglución?

2. ¿Cuáles de las diferentes consistencias para alimentar conoce usted?

3. ¿Qué signos o conductas del usuario considera usted como un problema de deglución? 3.1. ¿Usted considera la baja de peso como un problema de la deglución?

4. ¿Conoce alguna forma de proceder en caso de que el usuario se atore y no deje de hacerlo?

5. ¿Cuánto tiempo emplea en alimentar a cada usuario con problemas deglutorios?

6. ¿Tuvo alguna oportunidad de estudiar (o formarse) en temas relacionados con la deglución? ¿Cuáles? 
Tabla 3. Resumen de las preguntas de los grupos de discusión

\section{Preguntas}

1. ¿Tienen usuarios con problemas para tragar o comer?

1.1. Si es así, ¿de qué manera o qué tipo de atenciones se les da a aquellos que tienen o han tenido problemas para comer?

2. ¿Quiénes alimentan a los adultos mayores del centro?

3. ¿Cuáles de las diferentes consistencias para alimentar conocen ustedes?

3.1. ¿Le toca prepararlas a ustedes o a alguien más del centro?

4. ¿Cómo alimentan ustedes a los adultos mayores del centro?

5. ¿Qué acciones conocen para tratar a las personas con problemas deglutorios?

5.1. ¿Lo aprendieron en su formación o algún profesional les enseñó?

6. ¿Tuvieron alguna oportunidad de estudiar (o formarse) en temas relacionados con la deglución? ¿Cuáles?

7. De tener la oportunidad de recibir formación con un profesional especializado en el área de la alimentación en pacientes con alteraciones deglutorias, ¿qué consultas realizarían o que desearían aprender?

\section{Resultados}

— n este apartado se encuentran los antecedentes recopilados en el estudio, de los cuales se miento sobre la utilización de los diferentes tipos de consistencias, detección de problemas al alimentarse, manejo de problemas deglutorios, trabajo interdisciplinario y formación respecto a la deglución.

\section{Infravaloración de los trastornos deglutorios}

LOS TENS y los cuidadores relatan el problema de la alimentación como un síntoma o una característica del proceso de envejecimiento y no lo consideran un evento patológico. Esto se refiere al hecho de que si el adulto mayor presenta dificultades al alimentarse, no necesariamente se trataría de un trastorno de la deglución, pues estos son infravalorados. Ello se explica como un desconocimiento de los procesos fisiológicos o mecánicos cuando ocurre un trastorno deglutorio o problema de alimentación (no querer alimentarse) declarado por los propios participantes. En consecuencia, los TENS no identifican los signos de penetración laríngea como parte de un problema mayor de los residentes, calificados como una “dificultad para alimentarse”, ya que logran manejarlos con estrategias aprendidas en su quehacer, principalmente a través de modificaciones a las consistencias. No consideran estos eventos motivo de derivación para descartar una posible complicación deglutoria. Sumado a lo anterior, una vez el adulto mayor o residente con dificultad ha logrado alimentarse adecuadamente y se observa una “mejoría”, las TENS y cuidadoras declaran que deben avisar al comedor o nutricionista, para que exista un cambio de alimentación en ellos, ya que evidencian un proceso de superación de la dificultad. 
En otras palabras, ellos visualizan estos signos como una complicación en su quehacer, mas no como la posibilidad de una complicación mayor.

\section{Conocimiento sobre la utilización de los diferentes tipos de consistencias}

LOS TENS y los cuidadores utilizan la consistencia según sea indicado, en algunos casos por la nutricionista o el TENs con mayor conocimiento o experiencia. Al consultarlos por las diferentes consistencias de alimentación, nombran: normal, licuada, molida, blanda, entera, semimolida, líquida, néctar, papilla y líquido con espesantes. En la mayoría de los casos, los entrevistados indicaron que la consistencia normal no se debe emplear en los adultos mayores, a excepción de aquellos sin dificultades deglutorias declaradas. Además, manifiestan poco conocimiento acerca de las propiedades e identificación de cada consistencia, y no hay un consenso respecto a su uso. Los tens refieren que deben observar cómo se alimenta el adulto mayor e ir evaluando de forma intuitiva cómo reacciona a la nueva consistencia y, de ser necesario, modificarla nuevamente hasta encontrar la textura alimentaria adecuada. Indican que el licuado es para quienes generalmente están con bajo peso, aquellos sin dentadura y usuarios con dificultades para deglutir. En otros casos, existen adultos mayores que toleran la consistencia sólida, pero necesitan asistencia por parte de un auxiliar en enfermería para monitorear la forma en que se alimentan, ya que en ocasiones ingieren el alimento con mucha rapidez, y el tENs debe asistir y entregar él mismo, de forma pausada, monitoreando si existe atoro o no. En cuanto a la consistencia líquida, la utilizan en adultos mayores que comen semisólidos; se entrega

preferentemente a través de cucharas o bombillas, utilizando también esta consistencia en caso de atoros con sólidos. Se menciona, así mismo, que los líquidos son provistos ante la constante queja de los adultos mayores, quienes declaran sed constante y que están sometidos a dietas modificadas. Se nombra, además, la consistencia tipo néctar, entregada a los adultos mayores que sufren atoros con líquidos finos. Todos los manejos de consistencias, declaran, están establecidos por la nutricionista; el rol de los TENS y cuidadoras es informar a un profesional la dificultad de la alimentación para que este cambie la consistencia.

\section{Detección de problemas al alimentarse}

LOS TENS y cuidadores de los distintos centros señalan que detectan las dificultades de los adultos mayores en el momento de alimentarse, mayoritariamente cuando manifiestan signos de importancia, en especial cianosis, tanto en rostro como en uñas. Mencionan también la presencia de carraspeo y tos prolongada, es decir, cuando hay manifestaciones que ponen en riesgo la integridad física del usuario.

La mayoría de los cuidadores señala haber presenciado problemas de obstrucción de la vía aérea durante la alimentación con consistencias líquidas y semisólidas. Otra de las 
formas de detectar estas dificultades es a la vista: cuando observan una disminución de masa corporal importante que miden con el peso del usuario, principalmente cuando los adultos mayores son incapaces de comunicar sus problemas al comer, consecuencia en su mayoría de enfermedades neurodegenerativas.

Dentro del abordaje que se lleva a cabo luego de detectada la primera dificultad, los entrevistados declararon utilizar la maniobra de Heimlich. Ellos aluden a que es la única técnica que aprendieron en su formación. Posterior a esto, si no se observan mejoras inmediatas, recurren a un servicio de salud asistencial para que traslade a los adultos mayores. Finalmente, si existen serias complicaciones, mencionan la intervención de vías de alimentación alternativas, entre ellas la sonda nasogástrica, donde la mayoría reporta experiencia en el manejo de la sonda y la forma de alimentación hacia el usuario.

\section{Manejo de problemas deglutorios}

Frente al concepto del manejo de problemas deglutorios, la acción generalizada de los Eleam consiste en una evaluación previa al proceso de alimentación como tal, donde los dominios que se evalúan son: el tipo de utensilio que debe utilizar el adulto para comer, la consistencia con la cual se presenta el alimento, la forma en la que el adulto come, su postura dinámica durante la alimentación y una evaluación nutricional. Con los resultados obtenidos, el personal identifica si el usuario adulto mayor residente logra comer de forma autónoma, con supervisión de algún miembro del personal del establecimiento o si es totalmente dependiente de alguien para poder alimentarse de forma adecuada.

La causa aparente y generalizada de los problemas deglutorios de los usuarios que ingresan a los Eleam se debe principalmente a problemas neurodegenerativos o problemas cognitivos, que en muchas ocasiones generan impedimento de comunicar sus problemas para comer.

Al profundizar en el manejo de estas complicaciones, se identificaron tres grupos de opinión contextualizados: el primero lo constituyen aquellos quienes no conocen maniobras o ejercicios que se puedan aplicar a usuarios con problemas deglutorios. Dentro de estos, declaran que solo son observadores pasivos frente a otro profesional, como el fonoaudiólogo (cuando se ha posibilitado su intervención), y que ante la realización de alguna técnica que apunte a mejorar el problema deglutorio de algún usuario, optan por repetirlas en otros usuarios del Eleam. El segundo grupo lo constituyen aquellos quienes declaran manejar estos problemas cuando se presentan como un signo evidente (penetración o posible evento aspirativo). Las reacciones del grupo de TENS o cuidadores ante un evento aspirativo grave se llevan a cabo de cuatro formas: 1) aplicación de maniobra de Heimlich, que casi la totalidad de los entrevistados conoce (principalmente por imitación o experiencia laboral); 2) calmar al usuario, tranquilizándolo mientras se espera que el episodio remita; 3) solicitar apoyo kinesiológico (kinesioterapia respiratoria, consistente en masajes y técnicas de presión en el 
área pulmonar, sobre la caja torácica, para expulsar el alimento aspirado por el usuario) y 4) llamar a una ambulancia, en casos extremos. El tercer grupo (minoritario) menciona, en general, que llevan a cabo un trabajo preventivo de problemas deglutorios, el cual se centra en la generalización de ejercicios, técnicas y capacitaciones de cuidados clínicos que involucran al proceso deglutorio como tal. También logran adaptar cubiertos para la alimentación (como el grosor de su agarre), aplicar cambios de posición durante la alimentación, modificar consistencias, administrar maniobras compensatorias según el requerimiento del usuario y sentarlo a comer en la mesa. Todo esto apunta a mantener la calidad de vida de los usuarios en el Eleam. Estas técnicas, especifican, las adquirieron por la experiencia, por el consejo de algún otro profesional que se desenvuelve en el mismo establecimiento o por sentido común del personal que alimenta; no obstante, la minoría del personal que trabaja en un Eleam perteneciente a este grupo declaró adquirir este conocimiento de forma teórico-práctica durante su formación, ya sea como técnico profesional, técnico de nivel medio o por algún curso de capacitación específica.

Un aspecto escasamente abordado por profesionales y técnicos es el manejo ambiental frente a la deglución durante los procesos de alimentación cotidiana. Se concuerda que los adultos mayores comen todos de manera grupal en el mismo horario y espacio físico, lo que generaría distractores en los respectivos comedores que influenciarían en la atención del adulto, sobre todo en aquellos con alteraciones de base. Si bien se menciona la modificación ambiental de los dormitorios de los usuarios, no ocurre de la misma manera en los comedores. Además, se manifiesta que el tiempo para llevar a cabo el proceso de alimentación a los adultos mayores es insuficiente, debido a la alta cantidad de residentes por teNs en horarios de comida establecidos. Estos, en su mayoría son rígidos y de corta duración, los cuales son respetados, de manera de no entorpecer el funcionamiento organizacional de otras zonas de los Eleam.

\section{Trabajo interdisciplinario}

A través de las distintas entrevistas y grupos de discusión realizados en los centros de larga estadía, se evidenció por parte de los entrevistados la necesidad de contar con un profesional capacitado para abordar los problemas de deglución. Estos responden que se desconoce el rol del fonoaudiólogo como apoyo al manejo de estos usuarios; es patente un desconocimiento con respecto a su labor y profesión, pues en la mayoría de los casos el técnico profesional de enfermería debe solventar, en conjunto con cuidadores y técnicos de nivelación media, las diversas necesidades y problemáticas que surjan. Respecto a la participación de otros profesionales en dichas complicaciones, destacan la del kinesiólogo, el cual, de acuerdo con su opinión, interviene de manera reactiva ante una situación crítica generada por problemas deglutorios, y la del nutricionista, para identificar la consistencia más adecuada para los adultos mayores, sin reportar instancias de discusión entre disciplinas para casos específicos 
o la existencia de lineamientos claros consensuados entre los distintos profesionales que vayan en beneficio de los usuarios.

\section{Formación respecto a la deglución}

Dentro de los puntos explicitados durante las entrevistas y grupos de discusión, se constató la falta de una formación académica específica en el área de deglución, declarada por los entrevistados. Al consultar sobre el manejo de este concepto, los participantes evidenciaron poca claridad. La mayoría define el proceso de manera general como el acto de "tragar", sin mayores especificaciones o referencias. Señalan que no han recibido formación continua en el área.

Respecto a los conocimientos que manejan, indicaron que, en su gran mayoría, los adquirieron desde el mismo quehacer, a través de experiencias personales o de otros colegas. Finalmente, especificaron con menor frecuencia que algunos participantes han recibido formación profesional en el extranjero. Aun así, resulta visible, según los puntos expuestos durante las entrevistas y los grupos de discusión, la ausencia de contenidos elementales en la formación académica de los entrevistados en torno al área de la deglución y el proceso de alimentación. Son ellos mismos quienes indican que durante sus distintas instancias de formación no existió un profesional capacitado del área educando sobre la temática.

Ellos detectaron la necesidad de recibir información a través de charlas y capacitaciones que les aporten a sus competencias de trabajo y apunten a los cambios de posición, técnicas de alimentación, técnicas de estimulación de la deglución, prevención y detección de la penetración y aspiración en usuarios, pautas sobre los pasos frente a un atoro o aspiración, conocimientos sobre la detección temprana de la disfagia y educación a la familia sobre las dificultades en la alimentación.

\section{Discusión}

Ete es el primer estudio chileno sobre el conocimiento que poseen los profesionales y los
técnicos involucrados en la alimentación de los adultos mayores con posible disfagia o presbifagia.

Basándose en los datos recolectados por el personal entrevistado de cada Eleam, hay falta de conocimiento sobre cómo aplicar técnicas, maniobras, ejercicios, cambios posturales, entre otras, que potencien una deglución sana, segura, efectiva y contextualizada a los usuarios, lo cual es concordante con otros estudios internacionales (15-18). En cuanto a la detección, son capaces de reconocer si el adulto mayor residente presenta algún tipo de cambio cuando la manifestación es evidente y de importancia (cianosis, tos, carraspeo o falta de aire); pero la valoración y 
relevancia que se le da a este hallazgo que desencadena el problema deglutorio es infravalorado por parte del personal, lo que coincide con lo documentado tanto en el estudio de Kayser-Jones y Schell como en el de Pelletier (15,16). De este modo, es importante que los profesionales que se encuentran día a día con los adultos mayores se concienticen sobre esto, pues la prevención de las complicaciones deglutorias parte desde este punto. Un desconocimiento o infradetección de estas alteraciones puede inducir complicaciones mayores, como la neumonía aspirativa, que es potencialmente mortal en este grupo etario, consecuencia de la mantención de hábitos alimenticios no pertinentes para algunos adultos mayores (24).

Adicionalmente, el manejo de los problemas deglutorios ya detectados se da sobre todo en la modificación de consistencias, cambios de posición, adaptación de cubiertos, entre otros, que apuntan a la prevención de episodios de aspiración y posibilitan que el usuario se alimente de buena manera para mantener la funcionalidad y una buena calidad de vida; pero, en este caso, el proceso se aplica con base en la experiencia del personal y no por un sustento teórico-práctico (24). Ellos informaron que este no fue provisto durante su proceso formativo, y ello los haría caer en prácticas poco recomendadas o inseguras, por ejemplo, asignar semisólidos a aquellos adultos mayores que no los necesitan $(16,25)$. Se entiende que un profesional capacitado debe indicar la terapia y las medidas relativas a dichas dificultades; en este caso, el fonoaudiólogo, a través de una valoración detallada de cada persona (6,7). En ese sentido, tales modificaciones deberían hacerse en conjunto con ellos, quienes son los profesionales competentes para indicar, a través de una evaluación integral y un sustento en la evidencia, cuáles consistencias pueden ser ingeridas de manera segura y cuáles no, de manera personalizada $(26,27)$.

En este contexto, Chiang y Hwu, en un estudio en el que se reportaron las experiencias de auxiliares de enfermería, se evidenció que uno de los principales problemas de las participantes tuvo que ver con el manejo integral de la disfagia; se destacó la necesidad de contar con planes individualizados por cada usuario con algún tipo de trastorno e instrucciones claras sobre su proceder (18). En consecuencia, para ambos casos (detección y manejo) resulta necesario generar medidas educativas con el objetivo de mejorar estos aspectos.

Complementario a lo anterior, se destaca que, en gran medida, el manejo de estas alteraciones es sintomatológico y, en menor medida, focalizado en la detección o prevención, procesos que deberían sostenerse de manera interdisciplinaria $(7,28)$.

Pese a esto, la realidad es que estos establecimientos no siempre cuentan con fonoaudiólogos dentro de su equipo en salud. En este estudio, se informó que en algunos casos los familiares de usuarios residentes costean el servicio de estos profesionales de manera individual, a fin de mejorar o mantener el estado deglutorio del usuario afectado; pero la falta generalizada de ellos, como profesionales indicados en la evaluación y rehabilitación de los trastornos de la deglución, impacta en la comunicación, educación o decisiones relacionadas con el manejo, por lo que es una de las principales causales en la deficiencia 
tanto en proporción correcta de las consistencias como en el proceso de alimentación, que se evidencia además en la falta de tiempo de la alimentación, debido a que no se no se considera el rendimiento y capacidades de los usuarios, ni se aplican técnicas que apunten a una eficiencia deglutoria $(29,30)$.

El abordaje en general, evidenciado en este estudio cualitativo, parcialmente, coincide con el estudio de Rosales, quien buscó determinar el grado de manejo del paciente disfágico por parte de paramédicos en una unidad de cuidados intensivos (19). Si bien el contexto es diferente, los resultados de dicho estudio indicaron que el 61\% de ellos tuvo un manejo "no deseable”. En ese sentido, los resultados de la presente investigación pueden ser relevantes y transferibles a los diversos contextos donde estos profesionales se desempeñan con usuarios presbifágicos o disfágicos.

Lo descrito evidencia una clara necesidad por parte del personal involucrado en la alimentación de los adultos mayores del manejo de información respecto a cómo es y cómo se debe aplicar un proceso de alimentación sano, seguro y eficiente, sin perjudicar y potenciando la calidad de vida del adulto mayor residente (31).

Como fortalezas del presente estudio se destacan las propias del tipo de investigación. Los estudios cualitativos, a diferencia de los cuantitativos, son útiles para profundizar en experiencias, motivos o conocimientos que a menudo la cuantificación no provee (15). Se destacan, además, los contextos a los que el equipo de investigación pudo acceder, porque obtuvo una sistematización concordante de resultados para todas las instituciones. El acceso a la información y la experiencia de los participantes proveen un marco teórico de relevancia para visualizar la realidad del personal y su contexto, además de sus necesidades de educación, capacitación y comunicación.

Dentro de las limitaciones, se menciona la acotación de la investigación a comunas urbanas de la región de la Araucanía, aunque no por ello pierde relevancia, al ser el primer estudio nacional en su tipo. Adicionalmente, aquellas que son propias de su diseño, porque no se contemplaron las dimensiones procedimentales y habilidades blandas propias de las competencias asociadas al quehacer clínico. Se sugiere que, para futuras investigaciones, se contemple la estrategia de observación, para enriquecer los resultados.

A modo de síntesis, el manejo deglutorio de los adultos mayores en contexto de institucionalización constituye un proceso complejo. Los auxiliares de enfermería o cuidadores constituyen la primera línea de atención, al cumplir una gran cantidad de funciones. El hallazgo más importante de este estudio es la falta de conocimiento del personal sobre la deglución y sus complicaciones, además su manejo en este contexto. El abordaje de personas con posibles cambios o trastornos deglutorios debe ir de la mano de un adecuado conocimiento sobre la condición, sus elementos característicos y las estrategias terapéuticas, que deberían ser incluidas en programas de estudio propios de la disciplina o en formación continua. Paralelamente, resulta necesario que el proceso de toma de decisiones sobre la intervención 
se lleve a cabo de manera interdisciplinaria, para garantizar la pertinencia de las medidas y aumentar la seguridad tanto de los usuarios como de quienes les prestan cuidados.

Finalmente, los resultados descritos pueden orientar la generación de nuevas investigaciones, aportes o iniciativas basadas en las categorías obtenidas, que complementen la visión sobre el manejo deglutorio de los TENs, auxiliares y funcionarios de los Eleam, además de iniciativas interdisciplinarias que aborden esta problemática, en pos de mejorar la calidad de vida de la población de adultos mayores institucionalizados.

\section{Agradecimientos}

— ste proyecto de investigación fue posible gracias a las gestiones y cooperación brindada Mejores y Eleam Vicente de Paul. Agradecemos a los directores y directoras de estos establecimientos y a los funcionarios participantes del estudio.

\section{Contribución de los autores}

—elipe Henríquez: autor principal. Participa en la concepción y desarrollo de la idea de la inves— tigación, interpretación de los datos del estudio, revisión y análisis crítico del contenido intelectual. Aprobación final de la versión para publicar. Acuerdo para rendir cuentas de todos los aspectos del trabajo para asegurar que las preguntas relacionadas con la exactitud o integridad de cualquier parte del trabajo sean investigadas apropiadamente y resueltas.

Jordan Ferrufino: participa en la concepción y desarrollo de la idea de la investigación, interpretación de los datos del estudio, revisión y análisis crítico del contenido intelectual. Aprobación final de la versión para publicar. Acuerdo para rendir cuentas de todos los aspectos del trabajo, a efectos de asegurar que las preguntas relacionadas con la exactitud o integridad de cualquier parte del trabajo sean investigadas apropiadamente y resueltas.

Nicol Inzunza: participa en la concepción y desarrollo de la idea de la investigación, interpretación de los datos del estudio, revisión y análisis crítico del contenido intelectual. Aprobación final de la versión para publicar. Acuerdo para rendir cuentas de todos los aspectos del trabajo para asegurar que las preguntas relacionadas con la exactitud o integridad de cualquier parte del trabajo sean investigadas apropiadamente y resueltas.

Ana Ñancucheo: participa en la concepción y desarrollo de la idea de la investigación, interpretación de los datos del estudio, revisión y análisis crítico del contenido intelectual. Aprobación final de la versión para publicar. Acuerdo para rendir cuentas de todos los aspectos del trabajo 
para asegurar que las preguntas relacionadas con la exactitud o integridad de cualquier parte del trabajo sean investigadas apropiadamente y resueltas.

Eduardo Padilla: participa en la concepción y desarrollo de la idea de la investigación, interpretación de los datos del estudio, revisión y análisis crítico del contenido intelectual. Aprobación final de la versión para publicar. Acuerdo para rendir cuentas de todos los aspectos del trabajo para asegurar que las preguntas relacionadas con la exactitud o integridad de cualquier parte del trabajo sean investigadas apropiadamente y resueltas.

Valeska Soldan: participa en la concepción y desarrollo de la idea de la investigación, interpretación de los datos del estudio, revisión y análisis crítico del contenido intelectual. Aprobación final de la versión para publicar. Acuerdo para rendir cuentas de todos los aspectos del trabajo para asegurar que las preguntas relacionadas con la exactitud o integridad de cualquier parte del trabajo sean investigadas apropiadamente y resueltas.

Camilo Morales: participa en la concepción y desarrollo de la idea de la investigación, interpretación de los datos del estudio, revisión y análisis crítico del contenido intelectual. Aprobación final de la versión para publicar. Acuerdo para rendir cuentas de todos los aspectos del trabajo para asegurar que las preguntas relacionadas con la exactitud o integridad de cualquier parte del trabajo sean investigadas apropiadamente y resueltas.

\section{Descargos de responsabilidad}

os autores declaran no tener ningún conflicto de interés con instituciones o sectores inte-
resados en los resultados de esta investigación.

\section{Conflicto de intereses}

Ninguno declarado.

\section{Referencias}

1. Schindler JS, Kelly JH. Swallowing disorders in the elderly. Laryngoscope. 2002;112 (April):589-602. https://doi.org/10.1097/00005537-200204000-00001

2. González R, Bevilacqua JA. Disfagia en el paciente neurológico. Rev Hosp Clín Univ Chile. 2009;20(252):62.

3. Clavé P, Arreola V. Disfagia orofaríngea. Gastroenterol Hepatol Contin. 2010;9(6):267-74. https://doi.org/10.1016/S1578-1550(10)70056-5 
4. González C, Casado P, Gómez A, Pajares S, Dávila R, Barroso L, et al. Guía de nutrición para personas con disfagia [internet]. Madrid: Instituto de Mayores y Servicios Sociales; 2006. Disponible en: http://www.imserso.es/InterPresent2/groups/imserso/documents/ binario/402017002_guia_nutricion_perso.pdf

5. Clavé P. Disfagia orofaríngea en el anciano. Rev Esp Geriatr Gerontol. 2012;47(4):139-40. https://doi.org/10.1016/j.regg.2012.04.009

6. American Speech-Language-Hearing Association. Knowledge and skills needed by speech-language pathologists providing services to individuals with swallowing and/or feeding disorders. Vol. 22. 2002. https://doi.org/10.1044/policy.KS2004-00215

7. Payne MA, Morley JE. Dysphagia: a new geriatric syndrome. J Am Med Dir Assoc. 2017;18(7):555-7. https://doi.org/10.1016/j.jamda.2017.03.017

8. Decreto 14/2010, 24 de febrero. Aprueba reglamento de establecimientos de larga estadía para adultos mayores [internet]. Ministerio de Salud de Chile. Disponible en: http:// www.minsal.cl/sites/default/files/files/DTO-14_05-AGO-2010_ELEAM.pdf

9. Thumala D, Kennedy BK, Calvo E, Gonzalez-Billault C, Zitko P, Lillo P, et al. Aging and health policies in Chile: new agendas for research. Heal Syst Reform. 2017;3(4):253-60. https://doi.org/10.1080/23288604.2017.1353844

10. Ministerio de Salud, Subsecretaría de Redes Asistenciales de Chile. Decreto 59/2013 de 6 de septiembre, [internet]. Disponible en: https://www.leychile.cl/N?i=1057679\&f=2013$12-31 \& \mathrm{p}=$

11. Servicio Nacional del Adulto Mayor. Ministerio de Desarrollo Social. Estándares de calidad para establecimientos de larga estadía para adultos mayores. Protocolos Senama 2016. Santiago de Chile; 2016.

12. AIEP, Universidad Andrés Bello. Técnico en Enfermería Mención Geriatría [internet]. 2018. Disponible en: http://www.aiep.cl/mallasPDF/Tecnico_en_Enfermeria_Mencion_ Geriatria.pdf

13. Santo Tomás. Centro de Formación Técnica. Técnico en Enfermería y Procedimientos de apoyo a la Rehabilitación [internet]. 2018. Disponible en: http://ipcft.santotomas.cl/ carreras/tecnico-enfermeria-procedimientos-apoyo-la-rehabilitacion/

14. IPCHILE. Técnico en Enfermería con Mención en Geriatría [internet]. 2018. Disponible en: http://www.ipchile.cl/wp-content/uploads/2011/11/tecnico-enfermeria-mencion-geriartria.pdf

15. Kayser-Jones J, Schell E. The effect of staffing on the quality of care at mealtime. Nurs Outlook. 1997;45(2):64-72. https://doi.org/10.1016/S0029-6554(97)90081-6

16. Pelletier CA. What do certified nurse assistants actually know about dysphagia and feeding nursing home residents? Am J Speech-Language Pathol. 2004;13(May):99-113. https://doi.org/10.1044/1058-0360(2004/012)

17. Lea EJ, Goldberg LR, Price AD, Tierney LT, McInerney F. Staff awareness of food and fluid care needs for older people with dementia in residential care: a qualitative study. J Clin Nurs. 2017;26(23-24):5169-78. https://doi.org/10.1111/jocn.14066 
18. Chiang CK, Hwu YJ. Feeding experiences of nursing aides for residents with dysphagia. Geriatr Nurs (Minneap). 2018;39(4):436-42. https://doi.org/10.1016/j.gerinurse.2017.12.016

19. Rosales F. Manejo del paciente disfágico por técnicos paramédicos: evaluación y capacitación en un hospital chileno. Rev Chil Fonoaudiol. 2016;15:1-9. https://doi. org/10.5354/0719-4692.2016.44155

20. Strauss A, Corbin J. Basics of qualitative research. Vol. 3, Basics of qualitative research grounded theory procedures and techniques. Filadelfia: Sage; 2007.

21. Otzen T, Manterola C. Técnicas de muestreo sobre una población a estudio. Int J Morphol [internet]. 2017;35(1):227-32. Disponible en: http://www.scielo.cl/scielo.php?script=sci_arttext\&pid=S0717-95022017000100037\&lng=en\&nrm=iso\&tlng=en

22. Asociación Médica Mundial. Declaración de Helsinki de la AMM: principios éticos para las investigaciones médicas en seres humanos [internet]. 2013. Disponible en: https:// www.wma.net/es/policies-post/declaracion-de-helsinki-de-la-amm-principios-eticos-para-las-investigaciones-medicas-en-seres-humanos/

23. Ulin P, Robinson E, Tolley E. Investigación aplicada en salud pública: métodos cualitativos. Vol. 617. Washington D.C.: Organización Panamericana de la Salud; 2006.

24. Ekberg O, Hamdy S, Woisard V, Wuttge-Hannig A, Ortega P. Social and psychological burden of dysphagia: its impact on diagnosis and treatment. Dysphagia. 2002;17(2):13946. https://doi.org/10.1007/s00455-001-0113-5

25. Beattie E, O’Reilly M, Strange E, Franklin S, Isenring E. How much do residential aged care staff members know about the nutritional needs of residents? Int J Older People Nurs. 2014;9(1):54-64. https://doi.org/10.1111/opn.12016

26. Cichero JAY, Lam P, Steele CM, Hanson B, Chen J, Dantas RO, et al. Development of international terminology and definitions for texture-modified foods and thickened fluids used in dysphagia management: the IDDSI framework. Dysphagia. 2017;32(2):293-314. https://doi.org/10.1007/s00455-016-9758-y

27. Arteaga JP, Olavarria LC, Naranjo DB, Elgueta LF, Espinóla MD. Cómo realizar una evaluación de deglución completa, eficaz y en corto tiempo. Rev Otorrinolaringol Cir Cabeza Cuello. 2006;66(1). https://doi.org/10.4067/S0718-48162006000100003

28. Neumann V, Gutenbrunner C, Fialka-Moser V, Christodoulou N, Varela E, Giustini A, et al. Interdisciplinary team working in physical and rehabilitation medicine. J Rehabil Med. 2010;42(1):4-8. https://doi.org/10.2340/16501977-0483

29. Vega Y, Torres A, del Campo M. Análisis del rol del fonoaudiólogo(a) en el sector salud en Chile. Cienc Trab [internet]. 2017;59:76-80. Disponible en: http://www.scielo.cl/scielo.php?script=sci_abstract\&pid=S0718-24492017000200076\&lng=es\&nrm=iso\&tlng=es

30. Bleeckx D, Postlaux G. Deglución: evaluación y reeducación. EMC Kinesiterapia Med Física. 2002;23(2):1-11. https://doi.org/10.1016/S1293-2965(02)71935-X

31. Marín ZR. Elementos de nutricion humana. San José: Euned Universidad Estatal a Distancia; 1998. 